\title{
Improving image classifiers for small datasets by learning rate adaptations
}

\author{
Sourav Mishra \\ The University of Tokyo \\ 7-3-1 Hongo, Bunkyo-ku, Tokyo \\ sourav@ay-lab.org
}

\author{
Toshihiko Yamasaki \\ The University of Tokyo \\ 7-3-1 Hongo, Bunkyo-ku, Tokyo \\ yamasaki@ay-lab.org
}

\begin{abstract}
Our paper introduces an efficient combination of established techniques to improve classifier performance, in terms of accuracy and training time. We achieve two-fold to ten-fold speedup nearing state of the art accuracy, over different model architectures by dynamically tuning the learning rate. We find it especially beneficial in the case of a small dataset, where reliability of machine reasoning is lower. We validate our approach by comparing our method versus vanilla training on CIFAR-10. We also demonstrate its practical viability by implementing on an unbalanced corpus of diagnostic images.
\end{abstract}

\section{Introduction}

Image classification via deep learning has seen rapid improvements in the last few years. First introduced as a visual recognition challenge, its scope has permeated from industrial applications to medical uses [1. Various new architectures have been proposed since the introduction of AlexNet in 2012 [2], such as ResNet, DenseNet and Inception 3, 4, 5. These architectures have become the mainstay in most computer vision applications, as per current literature.

In addition to emergence of new architectures, we have also observed a steady increase in the accuracy of classification reported on standard datasets. However, this improvement cannot be solely attributed to the architectures alone. Learning paradigms such as newer loss functions, optimization methods such as Dropout [6], and DropBlock 7] along with better preprocessing routines have contributed to higher accuracy. However, some bottlenecks still remain. Conventional methods of training models require a large amount of data, often augmented or imputed from the original dataset to make it balanced. The phase of training also happens to be the longest task in the deep learning process. With the advent of cloud computing, much of the machine learning tasks have shifted to portals such as Amazon Web Services and Microsoft Azure. Larger architectures usually imply longer running tasks translating to higher billed costs. This is of paramount importance to research groups or startups working on limited time and financial resources to deliver outcomes.

To alleviate this training performance bottleneck, a large number of workarounds have been proposed over the years, albeit with lesser attention. In this paper, we have proposed an organic combination of existing techniques and some derivative ideas, focused on the hyperparameter of learning rate. We allow it to adapt predictably over the training phase to improve time (measured as wall time) as well as accuracy (measured as validation accuracy). Our empirical evaluations have led us to conclude that learning rate adaptation is an under-exploited resource in making a difference on the overall model performance.

In this paper we have presented the following:

- We investigated optimizing small dataset training by combining cosine rate annealing, cycle length multiplication and differential learning rates.

- We benchmark our scheme against conventional training paradigms, using CIFAR-10 dataset and quantify the speedup factor.

- We demonstrate the viability of this method on a corpus of dermatological diagnostic images which has an unbalanced nature.

Our paper is organized as follows. Following this Introduction we elaborate on the Methods in Section 2, describing the modus operandi of baseline determination, modified scheme and benchmarking. We present the results in Section 3 and demonstrate a practical application in Section 4. We conclude with Section 5 containing discussion \& inferences drawn. 


\section{Methods}

\subsection{Baseline Preparation}

For computing the baseline, we chose multi-class classification on the CIFAR-10 dataset 8. Our rationale of this choice was to select a data corpus reported commonly in contemporary literature, exhibiting sufficient variety and yet smaller than ImageNet or CIFAR100 1. Our classifier was built on PyTorch v0.4 framework with commonly recommended practices such as dynamic augmentation, early stopping and an option to resume training with a different (but fixed) learning rate. Pretrained ResNet-34, ResNet-50, ResNet-101, ResNet-152 and DenseNet-161 were chosen as the candidate architectures; a single GPU (NVIDIA Titan Xp 12 GB HBM2) was utilized for all the measurements. The batch size was set at 32 .

Prior to model learning, we normalized the data with the recommended mean $(0.4914,0.4822,0.4465)$ and standard deviation $(0.2023,0.1994,0.2010)$. The images were split in ratio of 5:1 into training and validation set. We performed dynamic in-memory augmentation by crop, horizontal \& vertical flips. Additionally we introduced other augmentation methods such as random-zoom by the imgaug package from Python repository.

All the models were trained to at least $90 \%$ validation accuracy for fair comparison. The training was commended with learning rate $\alpha=0.01$ and restarted manually with lower value $(\alpha=0.001)$, whenever early stopping forced the training to halt. Setting sufficient number of epochs to train was chosen based on prior experience. The accuracy of models and time to train are elucidated in Table 1.

\subsection{Finding Initial Learning Rate}

To optimize training performance, we focused on keeping learning rate $\alpha$ suitable throughout the model learning. Conventional wisdom dictates that learning rates should monotonically decrease during the course of training. However, starting with a value significantly smaller than theoretical optimum could lead to cost function never converging towards global minimum; similarly a larger value will lead to divergence or overfitting. It led to us to the pertinent question of determining an optimal initial learning rate. To accomplish this objective, we introduced an initial rate finder described by Smith et al. 9. The implementation used several mini-batches with gradually increasing values of $\alpha$, until the loss computed at end of each batch started decreasing dramatically. Finding the rate of change of loss, we could zero down to a good learning rate to begin. Figures 1 and 2 illustrate the learning rate range test for DenseNet-161.

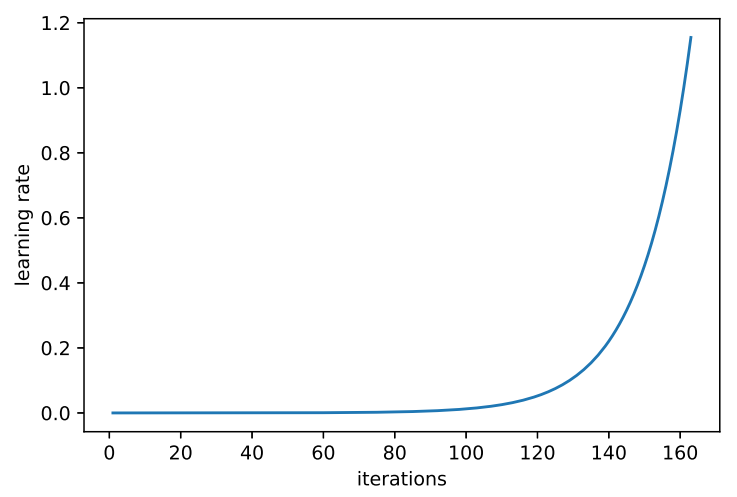

Figure 1: Determination of most suitable initial learning rate. The learning rate is systematically increased over a large range to determine where the losses start reducing.

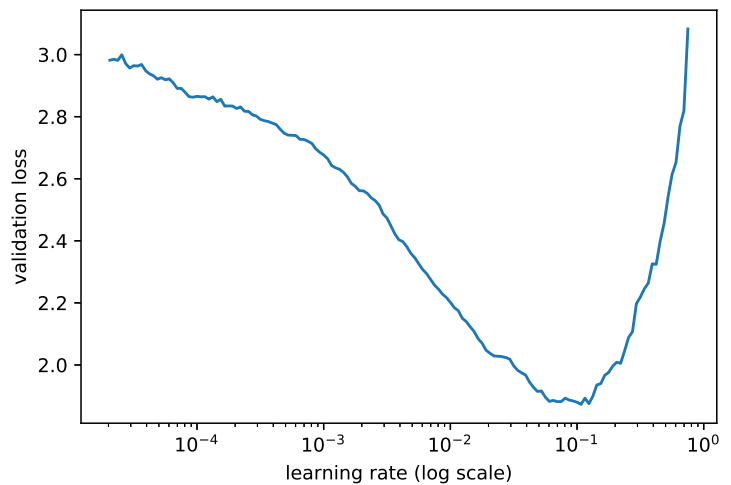

Figure 2: Plot of the losses measured over test minibatches to determine the optimum initial learning rate.

\subsection{Cosine Rate Annealing}

Following determination of the best initial rate, we chose to train the network with transforms similar to our baseline measurement. The differences we introduced at this stage were to employ L2 regularization and running a pass through our data to pre-compute activation values for the final layer, keeping all other layers frozen. As the first step, we froze all layers except the final layer to retain the complex features from ImageNet training. Initial shaping of the final layer promised performance advantages. Rather than keeping the learning rate fixed from what was determined, we adopted Stochastic Gradient Descent with Restarts (SGD-R), where cosine rate annealing gradually decreased the learning rate over the epoch from a designated value to zero [10. The scheduling is governed as shown in Equation 1: 


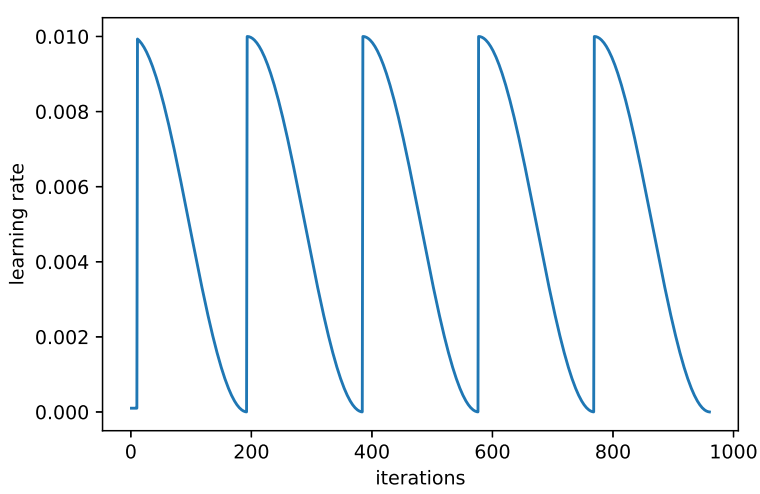

Figure 3: Plot of the cosine rate annealing over each epoch. The learning rate decreases from the precomputed optimal value to a minimum, over the course of epoch only to again restart with a new epoch.

$$
\nu_{t}=\frac{1}{2}\left(1+\nu \cos \left(\frac{t \pi}{T}\right)\right)+\nu_{\min }
$$

where $\nu$ is the initial learning rate, $t$ is the iteration over the epoch, and $T$ is the total number of iterations to cover a epoch. Up to 10 epochs were run using this method, until the validation accuracy stabilized. This scheduling operation is illustrated by Figure 3 for a training task carried out on DenseNet-161.

\subsection{Cycle Length Multiplication and Differential Learning Rates}

Our final step in the optimization scheme was to unfreeze all the network layers, and use differential learning rates for separate sections of the networks. Before diving into the rationale, we explain the following two concepts with brevity:

Differential Learning Rate (DLR) involved the process of assigning three separate learning rates, spanning the length of network \& adhering to cosine rate annealing over the cycle length.

Cycle Length Multiplication (CLM) involved extending the cosine annealing over progressively more integral number of epochs (with each subsequent cycle), as described in [10].

Initial layers of convolutional neural networks (CNN) typically capture rudimentary features, with complexity of the same increasing in later layers. More volatility can be envisioned in higher layer with each pass of stochastic gradient descent (SGD). Therefore assigning a very low-rate of $\alpha=0.0001$, left the initial layers virtually undisturbed, whereas a moderately high rate of $\alpha=0.01$ allowed elasticity for change in

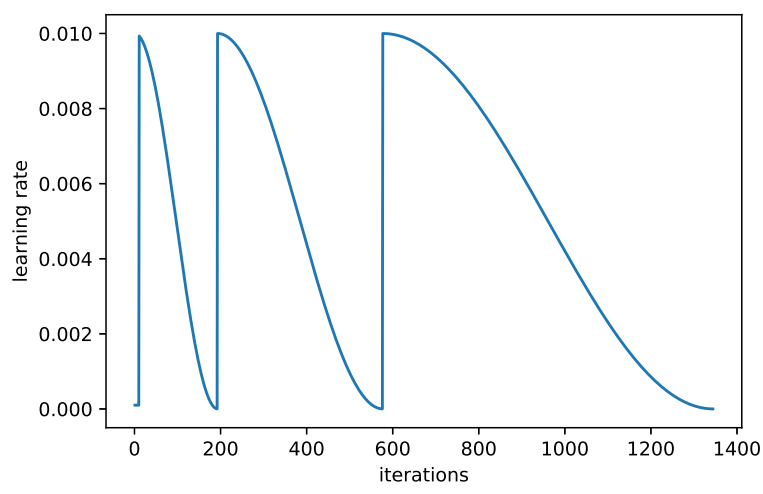

Figure 4: Cosine rate annealing extends to progressively higher number of epochs in cycle length multiplication.

the higher layers. The mid-section of the CNN was assigned a rate of $\alpha=0.001$.

Concurrent with changing the designated learning rates over different sections, we also extended the cycle length with a multiplication factor of 2 . As the model trained closer towards global minima, the parameters needed less perturbations. Gradually slowing down the SGD-R process guaranteed a better convergence towards global minima. The aforementioned step is illustrated by Figure 4, implemented on DenseNet161.

\section{Results}

The results from conventional training, described in Section 2.1, are illustrated in Table 1. For all the candidate architectures, early stopping halted model learning before empirically assigned maximum epochs. As stated previously, the training was resumed with $\alpha=0.001$, with a target to achieve a minimum of $90 \%$ validation accuracy. We have recorded learning duration and stable validation accuracy attained by early stopping with both the learning rates. Total time, which is a sum total of all learning durations, is presented in the last column.

The results from our optimization scheme are illustrated in Table 2. The columns highlight wall time recorded for phases described in Sections 2.2 through 2.4. The speedup factor on total time is indicated in the last column.

A confusion matrix for evaluation of the validation process on DenseNet-161 is presented in Figure 5. Matrices for other architectures are in the Appendix. We offer a few observations at this stage. We can see that models tune to near state-of-the-art accuracy values, without a high training time. Furthermore, they are capable of learning without over-fitting, on an orthodox choice of 5:1 training validation split of CIFAR-10. 
Table 1: Training on CIFAR-10 with conventional method

\begin{tabular}{c|cc|cc|c}
\hline \hline Model & Accuracy $(\alpha=0.01)$ & Time $(\mathrm{s})$ & Accuracy $(\alpha=0.001)$ & Time $(\mathrm{s})$ & Total time $(\mathrm{s})$ \\
\hline ResNet-34 & $86.25 \%$ & 14749 & $90.36 \%$ & 3008 & 17757 \\
ResNet-50 & $86.56 \%$ & 32596 & $90.54 \%$ & 1442 & 34039 \\
ResNet-101 & $86.35 \%$ & 58315 & $90.71 \%$ & 2323 & 60639 \\
ResNet-152 & $86.42 \%$ & 88520 & $90.68 \%$ & 3367 & 91888 \\
DenseNet-161 & $89.88 \%$ & 51109 & $93.02 \%$ & 3518 & 54628 \\
\hline \hline
\end{tabular}

Table 2: Training on CIFAR-10 with optimization scheme

\begin{tabular}{c|cc|cc|c|c}
\hline \hline Model & Acc. with SGD-R & Time (s) & Acc. with DLR+CLM & Time (s) & Total time & Speedup factor \\
\hline ResNet-34 & $82.45 \%$ & 3816 & $96.84 \%$ & 5840 & 9565 & 1.84 \\
ResNet-50 & $80.34 \%$ & 5345 & $96.82 \%$ & 6472 & 11817 & 2.88 \\
ResNet-101 & $84.20 \%$ & 2676 & $97.61 \%$ & 3998 & 6673 & 9.09 \\
ResNet-152 & $82.50 \%$ & 3517 & $97.78 \%$ & 5496 & 9012 & 10.20 \\
DenseNet-161 & $82.89 \%$ & 2001 & $97.15 \%$ & 5195 & 7195 & 7.59 \\
\hline \hline
\end{tabular}

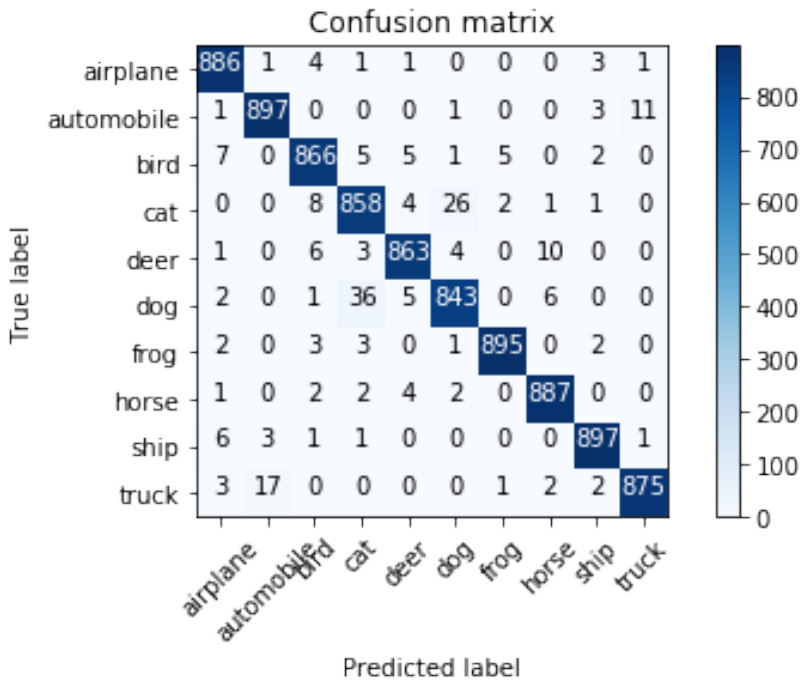

Figure 5: Confusion matrix for the quality of evaluation for CIFAR-10 on DenseNet-161

This demonstrates that learning rate is effective tool even with a smaller corpus of images. Most importantly, we observe that larger architecture receive better performance gains than smaller ones. We believe this is a valuable trait of our scheme, since current research is progressively moving towards using larger quantities of data and bigger architectures.

\section{Application}

To investigate benefits of our optimization technique and draw inferences, we introduce an application based on medical diagnostics for which this scheme was envi- sioned.

Dermatological diseases exhibit a wide variety in their manifestation. At a time when demand for medical consultation is rising, there is a severe under-supply of dermatologists in many countries. The number of practitioners in US has plateaued at 3.6 per 100,000 people and several East Asian countries advocate mobile clinics or tele-medicine [11. In the absence of immediate avenues, people resort to general practitioners (GP). Statistics indicate that the opinions of GPs are concurrent with dermatologists only $57 \%$ of the time 12. We attempt to provide machine learning based solutions to bridge this gap with a two-fold aim: To reduce workload of the dermatologist by aiding faster screening \& provide customized means to people for detecting possible skin problems

Most open medical image databases are small and unbalanced. Hence, there is a need to develop paradigms for effective training with limited information. Further, since disease labels could get updated periodically, or model requires customization for a new population type, training and deployment needs to be fairly rapid. Our schema manages to fulfill both the objectives, guaranteeing rapid deployment to the production servers. We chose a dermatological dataset with 10 unbalanced classes, containing 7543 images. The train-validation split was done unevenly to mimic a real database. We trained aforementioned architectures with vanilla scheme, followed by our optimization trick. We have compared the stable validation accuracy and total time across different architectures in Table 3. We again observed improved accuracy by around $10 \%$ points and speedup between 3.1 and 5.7 times. A confusion matrix based on ResNet-152 is shown in Figure 6. 
Table 3: Performance comparison between training schemes on dermatological images

\begin{tabular}{c|cc|cc|c}
\hline \hline Model & Accuracy (Conventional) & Time (s) & Accuracy (DLR+CLM) & Time (s) & Speedup \\
\hline ResNet-34 & $72.62 \%$ & 1576.33 & $83.40 \%$ & 343.20 & 4.6 \\
ResNet-50 & $71.88 \%$ & 2130.27 & $83.26 \%$ & 692.64 & 3.1 \\
ResNet-101 & $71.70 \%$ & 4216.22 & $83.19 \%$ & 1076.90 & 3.9 \\
ResNet-152 & $71.56 \%$ & 5437.72 & $84.90 \%$ & 958.88 & 5.7 \\
DenseNet-161 & $78.02 \%$ & 4156.75 & $84.61 \%$ & 946.14 & 4.4 \\
\hline \hline
\end{tabular}

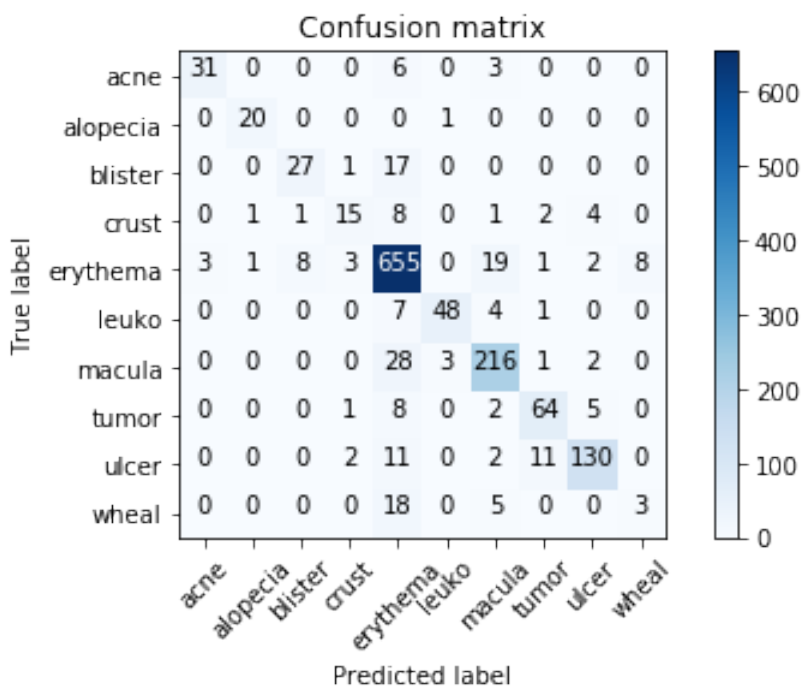

Figure 6: Confusion matrix for the quality of evaluation on dermatological dataset classified by learning on a pre-trained ResNet-152

\section{Conclusion}

We have demonstrated that there is much room for performance improvement in classical techniques for small datasets, by tuning the learning rate. It not only speeds up the convergence, but allows better model fits, increasing validation accuracy. By slowing down learning in initial layers of $\mathrm{CNN}$, it is easy to shift the bulk of computation towards the final layers, resulting in optimization of the process. Further, we observed that because of this combination of established techniques, models are more immune to learning bias in an unbalanced dataset, than traditional methods.

\section{Acknowledgement}

The authors offer their sincere thanks to D. Page (Myrtle AI) and FastAI community, who offered various tools \& resources for easy interpretation.

\section{References}

[1] Russakovsky, O., Deng, J., Su, H and Krause, J. "Imagenet large scale visual recognition challenge." International Journal of Computer Vision 115.3 pp.211-252, 2015

[2] Krizhevsky A., Sutskever, I and Hinton, GE. "Imagenet classification with deep convolutional neural networks". Advances in Neural Information Processing, pp. 1097-1105, 2012

[3] He, K., Zhang, X., Ren, S. and Sun, J. "Deep residual learning for image recognition." Proceedings of the IEEE Conference on Computer vision and Pattern Recognition, 2016.

[4] Huang, G., Liu, Z. and Van Der Maaten, L. "Densely connected convolutional networks." Proceedings of IEEE Conference on Computer Vision and Pattern Recognition, 2017.

[5] Szegedy, C., Liu,W., Jia, Y. and Sermanet, P. "Going deeper with convolutions." Proceedings of the IEEE Conference on Computer vision and Pattern recognition, 2015.

[6] Srivastava, N. and Hinton, GE. "Dropout: a simple way to prevent neural networks from overfitting." The Journal of Machine Learning Research, 15.1, pp. 19291958, 2014.

[7] Ghiasi, G., Lin, T.Y. and Q.V, Le. "DropBlock: A regularization method for Convolutional networks." Advances in Neural Information Processing Systems, 2018.

[8] Krizhevsky, A. and Hinton, GE." Learning multiple layers of features from tiny images". Vol.1 (4), Technical report, University of Toronto, 2009.

[9] Smith, LN. "Cyclical learning rates for training neural networks." 2017 IEEE Winter Conference on Applications of Computer Vision (WACV), 2017.

[10] Loshchilov, I. and Hutter, F. "SGDR: Stochastic gradient descent with warm restarts." Proceedings of the International Conference on Learning Representations (ICLR), 2017.

[11] Kimball, A.B. and Resneck, J.S. "The US dermatology workforce: a specialty remains in shortage". Journal of the American Academy of Dermatology, 59 (5), pp. 741-745, 2008.

[12] Lowell, B.A., Froelich, C.W., Federman, D.G. and Kirsner, R.S., "Dermatology in primary care: prevalence and patient disposition". Journal of the American Academy of Dermatology, 45 (2), pp. 250-255, 2001. 


\section{Appendix}

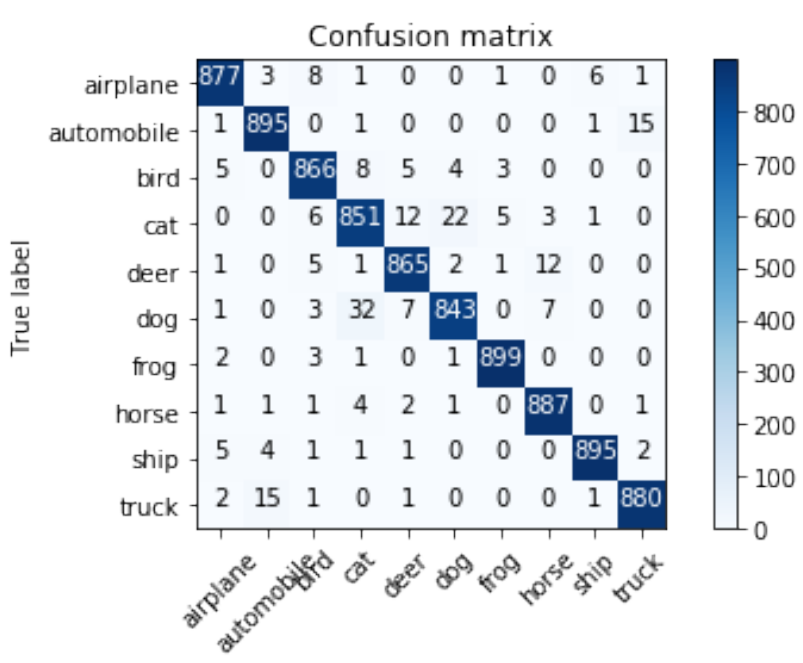

(a) ResNet-34

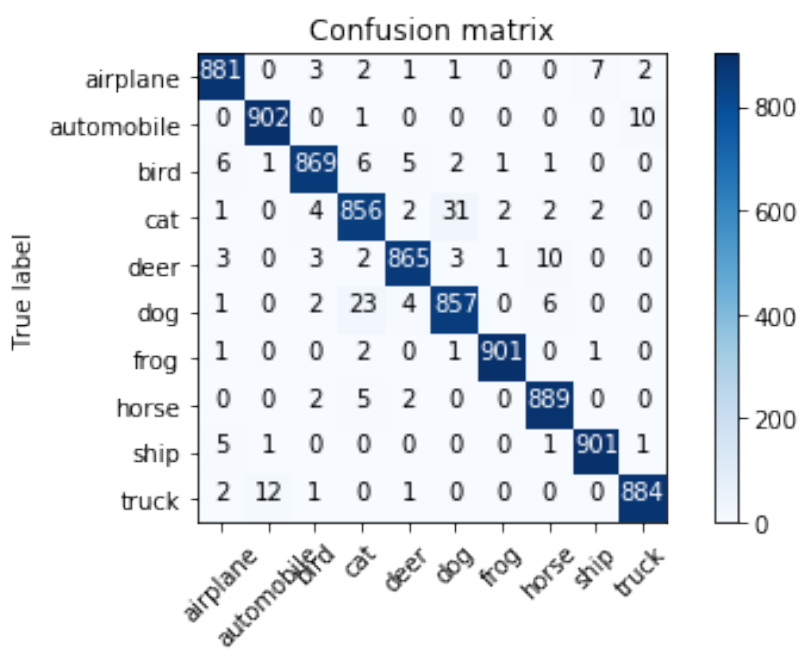

Predicted label

(c) ResNet-101

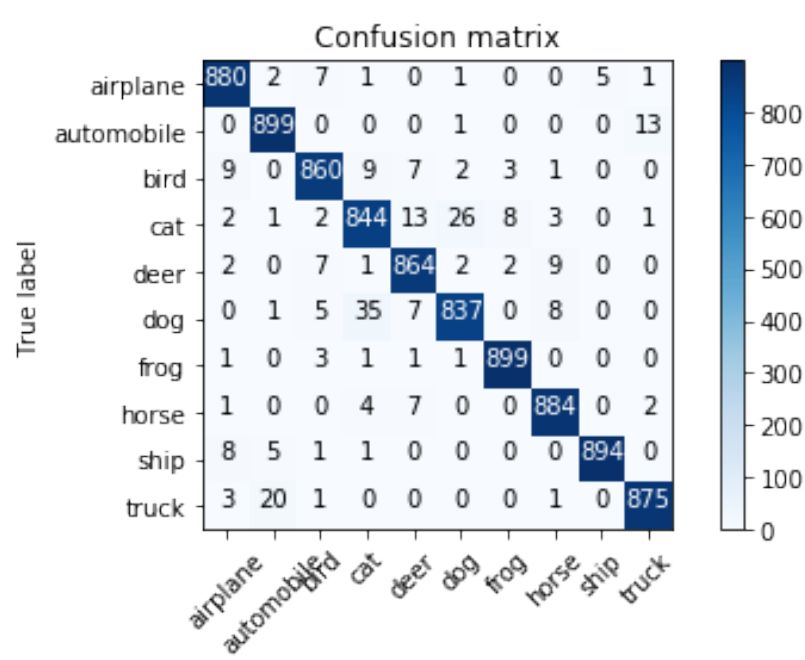

Predicted label

(b) ResNet-50

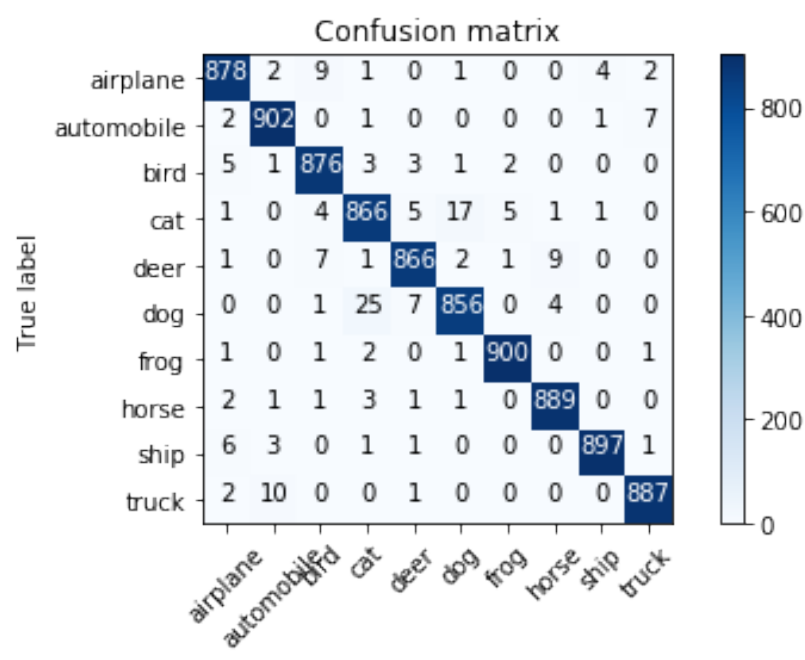

Predicted label

(d) ResNet-152

Confusion matrices of validation set from different ResNet architectures trained on CIFAR-10 dataset 